\title{
Education in care ethics: a way to increase palliative care awareness in India
}

\author{
Joris Gielen ${ }^{1}$
}

Published online: 2 September 2015

(C) Springer International Publishing AG 2015

\begin{abstract}
In India, the private healthcare sector is rapidly growing. The focus on profit and curative treatment in this sector carries the danger of overtreatment and lack of attention to types of care where the margin of profit is limited, such as palliative care. Since further expansion of the private healthcare sector is unavoidable and even necessary due to limited government spending on healthcare in India, ways to promote palliative care awareness in such an environment need to be found. An important step towards this goal is the development of healthcare ethics education with a focus on those ethical theories which are most appropriate from a socio-cultural perspective. Principlism, the dominant model in Western healthcare ethics which applies the principles autonomy, beneficence, nonmaleficence and justice to ethical cases, may not be most suited to the Indian context. Principlism very strongly focuses on individualistic autonomy. Although autonomy can obviously not be neglected in Indian healthcare, ethical theories which pay more attention to relational aspects may be more appropriate. In this context, Care Ethics appears as a valuable ethical theory. Moreover, the focus on caring relationships in Care Ethics clearly points to the need of palliative care. Development of socio-culturally appropriate healthcare ethics education can inculcate ethical sensitivity which will benefit palliative care in India.
\end{abstract}

Keywords Care ethics $\cdot$ Palliative care $\cdot$ India $\cdot$ Principlism

\section{Introduction}

In Indian healthcare, the government plays an important, but limited, role. This is mainly because of the increasing involvement of the private sector in healthcare. Over the course of the last two decades, healthcare contributions from the private sector have

Joris Gielen

gielenj@duq.edu

1 Center for Healthcare Ethics, 301A Fisher Hall, 600 Forbes Avenue, Pittsburgh, PA 15282, USA 
grown tremendously. Between 2002 and 2010, the private sector created more than $70 \%$ of new hospital beds, eventually representing $63 \%$ of all beds by 2010 (Gudwani et al. 2012). In the coming years, the share of the private sector is expected to grow even further with India's economic expansion. India's economic development has increased disposable family incomes, and this has allowed people to spend more money on healthcare, which has further resulted in a higher demand for healthcare. However, government health expenditure has remained low. In 2012, it stood at a meager $1.3 \%$ of GDP (World Bank 2015). This means that there are many opportunities for the expansion of the private healthcare sector. This expansion is not only inevitable, but in the current scenario of limited government spending on health care, it is even necessary in order to provide sufficient medical care to India's growing and increasingly affluent population.

Leaving a substantial share of healthcare in the hands of the private sector also includes specific risks, though. If private healthcare initiatives are not driven by a specifically articulated humane vision, they may develop a tendency to have profit making as their primary goal. Although profit making is not a problem in itself, it may cause healthcare providers to focus too narrowly on those types of care which maximize profit. This engenders two possible dangers. The first danger is overtreatment and the administration of futile treatment. Invasive, burdensome, and expensive treatments with little or no chance of benefit to the patient are administered due to the financial gain they bring to the institutions or healthcare providers involved in their administration. The second is the danger of marginalization of those types of care whose margin of profit is limited. This may be one of the factors that can explain the limited response palliative care has generated among healthcare providers in India over the last two decades.

Palliative care was introduced in India in the second half of the 1980's. Since then, palliative-care programs have been developed in different parts of India, although coverage of palliative care in India still remains a huge problem. At present, palliative-care services are functioning in just over half of the Indian states (Indian Journal of Palliative Care 2015).

This leaves a very paradoxical situation. On the one hand, further expansion of the private healthcare sector is inevitable and even necessary. On the other hand, this evolution poses certain risks to patient wellbeing. Can a way out of this paradoxical situation be found? People involved in palliative care can point out the need for promotion of palliative care awareness in the private healthcare sector. In cases of advanced disease, palliative care awareness will make healthcare providers realize that there are important treatment choices to be made that have a tremendous impact on patient wellbeing and quality of life. For example, curative treatment may very often not be the preferred option. Increased palliative care awareness will obviously not solve all the issues related to the involvement of the private sector in Indian healthcare. However, at the very least it will lead to more appropriate attention to and treatment of the physical, social, psychological, and spiritual burdens associated with advanced disease.

Direct education in palliative care is an important part of this promotion of palliative care awareness, yet palliative care education as such is not enough. Palliative care awareness implies a change in attitude: a different way of looking at healthcare. Healthcare providers need to interiorize the deeper human values which lie at the basis of healthcare. Such interiorization can be an antidote to blind profit making, as well as a 
narrow focus on cure, and it should be one of the goals of good education in Indian healthcare ethics. This is not to say that healthcare providers who have not had access to this kind of education are immoral. Even when healthcare providers administer futile treatment to a terminally ill patient and make a handsome profit, they may ultimately decide to administer the treatment due to a lack of palliative care awareness and knowledge of viable options. They may feel helpless in front of the patient's suffering and honestly believe that the futile treatment is the only thing that they can offer the patient. Through education in healthcare ethics, they may learn the ways to critically assess such ethical cases in order to contribute to decisions that are in line with the ethical ideals of palliative care.

In order to further explore the possible contributions of education in healthcare ethics to the promotion of palliative care awareness in India, we need to address the following three questions.

- Can healthcare ethics education really make a difference in India?

- What is the most effective way to teach healthcare ethics in India?

- How will palliative care benefit from such ethics education?

In the following sections we will search for an answer to these questions.

\section{Healthcare ethics in medical education in India}

At present, the answer to the question of whether or not healthcare ethics education can make a difference in India can only be a hypothetical one. Healthcare ethics education in India is making progress, but it is still in its infancy. A recent mapping exercise of teaching of public health ethics revealed that little attention is paid to ethics training in medical education (Pati et al. 2014). In medical and nursing curricula, some attention is often given to healthcare ethics, but this is not always the case and does not seem to properly prepare the students for the ethical difficulties that they will encounter in medical and nursing practice.

A recent study among 111 postgraduate medical students in South India found that only $26.1 \%$ had acquired knowledge of bioethics during training. A stunning $75.7 \%$ was of the opinion that knowledge of ethics was of little to no importance in their work (Janakiram and Gardens 2014). A study in a government hospital in Chennai among 51 treating physicians and 58 other non-physician service providers on knowledge and practice of clinical ethics found that these medical professionals needed more ethics education (Subramanian et al. 2013). These were two small-scale studies, and the results cannot necessarily be projected to all Indian postgraduate medical students or medical professionals. However, they are telling nonetheless. The lack of training and insight in ethics is a serious obstacle to the further development of palliative care in India, because it indicates that many healthcare providers are insufficiently made aware of ethical complexities in healthcare, such as those encountered in advanced diseases. Further, they may be ill prepared to question healthcare practices that uniquely focus on cure at the expense of care, and they may not see the need for palliative care.

Moreover, evidence from other countries suggests that ethics education and integration of ethics consultations in medical decision making do influence healthcare 
providers' practices and patient outcomes. A study conducted in the US among nurses and social workers found that participants who had had more ethics education and training felt more confident in their moral judgments and were more likely to use ethics resources, such as ethics consultations, and take moral action (Grady et al. 2008). That study only analyzed how nurses and social workers perceived their actions and did not assess real actions or effects on patient outcomes. These outcomes were studied in a prospective, multicenter, randomized controlled trial conducted by Scheiderman et al. from November 2000 to December 2002 in the US. 551 patients in whom the researchers observed value-laden treatment conflicts, such as disagreement regarding forgoing life-sustaining treatment, were randomly assigned either to usual care $(n=273)$ or an intervention $(n=278)$. In the latter case, an ethics consultation was offered. There were no differences in mortality between both groups, but the researchers did observe significant reductions in hospital and ICU days and life-sustaining treatments in the intervention group (Schneiderman et al. 2003).

Admittedly, we are still far removed from the systematic integration of ethics consultations in medical decision making in India. However, the results of the Schneiderman study do show that involvement of professionals with a solid background in ethics does make a difference to patients, especially those who are at the end of their lives and may benefit from palliative care. Offering adequate ethics training to healthcare providers in India is an important first step towards the involvement of people with a solid ethics background in medical decision making processes. What is more, the observations of Schneiderman et al. indicate that even in the private healthcare sector in India involvement of people adequately qualified in healthcare ethics may lead to the administration of less futile treatment at the end of life. Since in many cases people educated in healthcare ethics will propose palliative care alternatives instead of the forgoing of treatment, there is a clear indication that education in healthcare ethics will lead to palliative care awareness, which has a profound impact on medical decision making.

\section{Teaching socio-culturally appropriate ethical theories}

If substantial benefits of healthcare ethics education to the development of palliative care awareness can be expected, we still need to determine the most fruitful approach to healthcare ethics education in India. In this regard, it may be tempting to have a look at the West, where healthcare ethics education has been better established, and copy their dominant ethical models and theories into the Indian medical syllabi and CME's. A model which is very prominent in Western healthcare ethics is Principlism. This wellknown ethical framework applies the principles of autonomy, beneficence, nonmaleficence and justice to ethical cases (Beauchamp and Childress 2009). However, we should wonder if the prominence of this model in the West is sufficient to prefer it over other theories when assessing ethical cases in Indian healthcare or the teaching of bioethics in India. Principlism has been developed in the West, and, consequently, the historical and cultural context from which it was generated has decisively shaped the formulation and interpretation of the principles. This could make the application of Principlism outside the Western culture questionable and dubious. This becomes very clear when we analyze the principle of autonomy. 
It can be argued that within Principlism the concept of autonomy is very important and more developed than the other principles. In Western bioethical discussions this principle is very often interpreted in an individualistic way. Generally, it is not recognized that such an interpretation is largely contingent upon a specifically Western historical and socio-cultural context and that other interpretations of autonomy are even possible. This emphasis on an individualistic autonomy is the logical outcome of Western ethical theories, which largely "built on the image of the independent, autonomous rational individual" (Held 2006, 10). Consequently, in Western societies there is such a strong focus on the ideal of the autonomous individual that it has been driving ethical debates, even including discussions on the legalization of voluntary euthanasia, i.e., "the intentional administration of lethal drugs in order to painlessly terminate the life of a patient suffering from an incurable condition deemed unbearable by the patient, at this patient's request" (Broeckaert 2008, 2009a; c; Broeckaert and Flemish Palliative Care Federation 2006). The ideal of "the independent, autonomous rational individual" has been effectively instrumental in the legalization of voluntary euthanasia in the Netherlands, Belgium, and Luxembourg. The reasoning is that if a terminally ill autonomous patient considers his or her suffering unbearable and wants to die, the dying wish can be granted due to it being the will of a rational individual. This is not to say that other principles and values were of no importance in the societal and political debates that led to this legislation. For instance, it can be argued that the principle of beneficence also played a role. In the euthanasia laws in the Netherlands, and also in Belgium and Luxembourg, it is stipulated that physicians are part of the decision making process. They have to be convinced that euthanasia is the best and only option for the patient (Widdershoven 2002). Yet, in the end the focus is very much on the wishes of the individual patient, and the views of other people involved in their care, such as nurses, family, and friends, do not receive substantial attention.

The focus on the autonomous individual can also be seen in the Death with Dignity Act of the American state of Oregon. This act does not allow for euthanasia, but it enables competent terminally ill patients to opt for physician assisted suicide, i.e., a physician who intentionally assists "a patient, at this patient's request, to terminate his or her life" (Broeckaert 2008, $2009 \mathrm{a}$; c; Broeckaert and Flemish Palliative Care Federation 2006). Although the Act does demand that "the attending physician shall recommend that the patient notify the next of kin of his or her request" for assisted suicide, the patient is under no obligation to yield this recommendation while still remaining eligible for assisted suicide (Oregon Health Authority 2015).

This kind of legislation would be unthinkable in countries such as India where the family and the treating physician play a much larger role in the medical decision making process than in the West (Raj 2012; Seetharam and Zanotti 2009; Senarath and Gunawardena 2009; Chattopadhyay and Simon 2008; Chaturvedi 2008). Partially, this intervention of others in decision making can be explained by paternalism. From the perspective of Indian physicians, there is undeniably a strong inclination towards physician paternalism. For instance, a study conducted among 48 physicians practicing in a tertiary hospital in Srinagar (Kashmir, India) showed that they were very selective in disclosing medical information to patients and would not respect a patient's refusal of treatment if they were of the opinion that the intervention would save their life (Yousuf et al. 2007). 
Yet, patients' involvement in medical decision making in India is determined by more factors than just the paternalistic attitude of physicians. Healthcare decisions in India are taken in a larger complex of relationships than in Western countries. People tend to get involved in the decisions driven by concern not only for the patient but also for all other people who may be affected by the treatment decision. Family members of patients often withhold medical information from a patient with advanced disease, because they are afraid that knowledge about the diagnosis and prognosis will destroy the patient's hope and in this way accelerate the disease progress or complicate emotional wellbeing (Chattopadhyay and Simon 2008; Raj 2012). But oftentimes, when treatment decisions are made the wellbeing of the family is also at stake. Due to a lack of health insurance, most healthcare spending in India is out-of-pocket. Therefore, a terminal illness of a family member can literally ruin a family's finances, particularly when the breadwinner is struck by the disease (Chattopadhyay and Simon 2008). In such a context, involvement of the family in the decision making process is much more obvious.

This is not to say that the principle of autonomy can be neglected in Indian healthcare and healthcare ethics education. In India, many patients are not satisfied with their limited opportunities to participate in medical decision making and actively want more information regarding their disease (Raj 2012; Raja 2007). Out of 100 patients who were admitted to the department of gastrointestinal surgery at a tertiary hospital in New Delhi, 98 wanted information regarding diagnosis and treatment options (Sanwal et al. 1996). This shows that there are no excuses for neglecting patient autonomy in Indian healthcare and healthcare ethics education. However, the particular individualistic interpretation of autonomy that permeates through many ethical models may not be most suited to the Indian context. Ethical theories which pay more attention to relational aspects may be more appropriate to Indian healthcare and healthcare ethics education in India. In this context, Care Ethics appears to be the most valuable ethical theory towards this aim.

\section{Care ethics in Indian culture}

Originating in the 1980's, Care Ethics was formed from the insight of feminist philosophers that most ethical theories had failed to account for the particular experience of women. In their view, ethical theories tended to focus on the rational autonomous individual, who was directly or indirectly supposed to be male. The feminist philosophers argued that, whether by nature or nurture, women's perspective on ethics is determined by their caring attitudes and practices. At the time, this was a major insight, because within ethical debates attention to the ethical dimensions of daily caring and mothering was virtually absent (Held 2006, 26-28, Groenhout 2004, 14 18). Care ethicists used that insight to argue that the autonomous individual rationally deliberating upon his future is not central to human existence. This is most obvious in the case of small children or elderly patients, who often entirely depend on others. However, without realizing it, to some degree all humans are dependent on others and need some kind of care. In fact, according to care ethicists, care is truly central to human lives (Groenhout 2004, 9-10, 33-35). 
Because everyone must rely on others to fulfill their caring needs, people are intimately connected. In Care Ethics, there is a strong sense of "human interdependence" (Tronto 1993, 101). People depend on each other for care. That is why Care Ethics focuses on what Ruth E. Groenhout has called the "ideal of human lives as grounded in a web of care with other people" (Groenhout 2004, 24). The fact that all human beings are interconnected has lead care ethicists to the conclusion that no one ever is fully independent, and individualistic autonomy seems to be an unrealistic ideal (Tronto 1993, 162-163).

In more recent works, care ethicists have repeatedly emphasized that the interdependence through care is a universal reality. Care is central to human existence anywhere (Groenhout 2004, 9-10). Thus, although Care Ethics originated from the desire of Western feminists to provide a space for the care experiences of women within ethical discourse, its proponents argue that its implications are valid for women and men no matter where they live. In this way, Care Ethics provides a normative framework that can claim transcultural validity (Held 2006, 46). From that perspective it may be a useful theory in the Indian socio-cultural context.

Indeed, in Indian society the ideal and centrality of care is very often clearly articulated. By custom and social pressure, people are called to care not only for their children, but also for their elderly parents and even for more distant relatives who have been less fortunate in life. It is true that the contemporary reality of urban life with both spouses having full-time jobs or living abroad often makes it difficult to comply with this ideal and properly care for their elderly parents or other relatives with needs. However, this does not seem to have really affected the importance of care as an ideal. Consequently, people who do not fulfill their caring duty are frowned upon with both men and women being blamed in equal measure for the plight of their relatives or parents. The close and decisive involvement of the family in the medical decision making process in India should be seen as a logical consequence of that ideal. People feel that they are called to care, and they are willing to take up that responsibility in the case of the illness of a family member or relative. Also, when they try to hide a poor prognosis from a terminally ill relative this should be seen as a sincere, albeit most often misguided, expression of the ideal of care. Driven by their willingness to care, they hide the diagnosis hoping that this will protect the patient from emotional harm.

The ideal of care is also ingrained within Indian philosophical reflection upon society. This can be illustrated by the way in which Hindu reformers have interpreted caste in the first half of the twentieth century. In Western literature, caste is often represented as a system of systematic oppression. However, besides the fact that Western representations of this extremely complex phenomenon tend to be simplistic and stereotypical, they are also far removed from how reformers such as Mohandas Karamchand Gandhi and Sarvepalli Radhakrishnan saw it. In their view, caste divided society in groups which had specific functions in society. Because these groups needed and depended upon each other, priests, politicians, soldiers, traders, craftsmen and farmers were considered equally important. Society could only survive and thrive if all castes performed their specific tasks in a spirit of mutual interdependence and care for each other (Gielen 2013, 85-89).

Gandhi and Radhakrishnan recognized that the relationships between castes were not always fully equal and that persons do not choose the caste to which they belong, 
since a person's caste is determined by birth. Yet, this does not make their observations irrelevant from a Care Ethics perspective. To the contrary, as Verginia Held argued:

The ethics of care addresses rather than neglects moral issues arising in relations among the unequal and dependent, relations that are often laden with emotion and involuntary, and then notices how often these attributes apply not only in the household but in the wider society as well. For instance, persons do not choose which gender, racial, class, ethnic, religious, national, or cultural groups to be brought up in, yet these sort of ties may be important aspects of who they are and how their experience can contribute to moral understanding (Held 2006, 13).

Undoubtedly, the ideas of Gandhi and Radhakrishnan regarding caste were somewhat naïve, and it is very unlikely that their representation of caste has been or will ever be a reality. But their insights do show how the ideals of care and interdependence are a part of Indian culture. There may be various reasons why healthcare ethics has not received the attention that it deserves in medical education. Could the lack of interest in bioethics education in India also be attributed to the inappropriateness of the dominant Western models to the Indian context? If this is the case and we accept that Care Ethics may be closer to India's socio-cultural reality, teaching models based on Care Ethics may stimulate interest in healthcare ethics among medical professionals in India.

There is one more reason why Care Ethics should be integrated in healthcare ethics education in India. Application of Care Ethics to medical cases will often lead to palliative care treatment. And, in this sense, it can be said that education in Care Ethics contributes to palliative care awareness. This requires some explanation. Care Ethics is not an abstract ethical theory. In Care Ethics, the importance of care practices are strongly emphasized. When confronted with a person who needs care, inaction is not an option for a caring person: action is required (see e.g., Tronto 1993, 102-105, 108-109, Held 2006, 57).

In the healthcare context this implies that when curative treatment is no longer effective, futile treatment cannot be administered, because it goes against the patients' care needs. At the same time, it is generally not possible to leave patients at their own devices without professional care. Something has to be offered. If we agree with Joan C. Tronto that caring involves "taking the concerns and needs of the other as the basis for action" (Tronto 1993, 105) it not only becomes clear that terminally ill patients do have concerns and needs which demand caring action, but that the holistic approach of palliative care will appropriately cover many of these concerns and needs. Palliative care indeed corresponds very well to Tronto's "four elements of care": attentiveness, responsibility, competence, and responsiveness (Tronto 1993, 127-137).

Attentiveness implies awareness of needs which require care. The members of interdisciplinary palliative care teams are trained to develop attentiveness for the physical, psychological, social, and spiritual needs of patients and their relatives. As professional caregivers, the members of the palliative care team also take up responsibility for the needs of patients who have been entrusted into their care. Due to continuing education, they have the competence to provide appropriate care. The care-receiving patient will somehow respond to the care, which enables the palliative care providers to reflect and act upon the patient's vulnerability and their own care needs. In fact, the link between Care Ethics and palliative care is rather obvious and does not demand further elaboration. Yet, it is still important to emphasize this 
connection within the context of healthcare ethics education in India, because it will nurture much needed palliative care awareness.

\section{Conclusion}

It is clear that further development of healthcare ethics education in India is urgently required. This education should be more than a quick introduction to some important issues in healthcare ethics, and it should teach healthcare providers a different way of looking at healthcare and the patients they are treating. In India, this can be achieved through the integration of a Care Ethics perspective in healthcare ethics education. Such socio-culturally appropriate healthcare ethics education can inculcate ethical sensitivity, which will benefit palliative care in India.

\section{References}

Beauchamp, T.L., and J.F. Childress. 2009. Principles of biomedical ethics. New York: Oxford University Press.

Broeckaert, B. 2008. Treatment decisions at the end of life: a conceptual framework. In Palliative care nursing. Principles and evidence for practice, ed. S. Payne, J. Seymour, and C. Ingleton, 402-421. Birkshire: Open University Press.

Broeckaert, B. 2009a. Euthanasia and physician assisted suicide. In Palliative medicine, ed. D. Walsh, 110115. Philadelphia: Elsevier.

Broeckaert, B. 2009b. Treatment decisions in advanced disease. A conceptual framework. Indian Journal of Palliative Care 15(1): 30-36.

Broeckaert, B., and Flemish Palliative Care Federation. 2006. "End of Life Decisions. A Conceptual Framework.” Accessed 6 April 2015. http://www.palliatief.be/.

Chattopadhyay, S., and A. Simon. 2008. East meets West: cross-cultural perspective in end-of-life decision making from Indian and German viewpoints. Medicine, Health Care and Philosophy 11(2): 165-74.

Chaturvedi, S.K. 2008. Ethical dilemmas in palliative care in traditional developing societies, with special reference to the Indian setting. Journal of Medical Ethics 34(8): 611-5.

Gielen, J. 2013. God in India. Diversiteit en devotie in de Indische religies. Tielt: LannooCampus.

Grady, C., M. Danis, K.L. Soeken, P. O’Donnell, C. Taylor, A. Farrar, and C.M. Ulrich. 2008. Does ethics education influence the moral action of practicing nurses and social workers? The American journal of bioethics : $A J O B$ 8(4): 4-11.

Groenhout, R.E. 2004. Connected lives: human nature and an ethics of care. New York: Rowan and Littlefield.

Gudwani, A., P. Mitra, A. Puri, and M. Vaidya. 2012. India Healthcare: inspiring Possibilities, Challenging Journey. Accessed 22 January 2015. http://www.mckinsey.com/.

Held, V. 2006. The ethics of care. Personal, political, and global. Oxford: Oxford University Press.

Indian Journal of Palliative Care. 2015. "Palliative Care Programmes by State.” Accessed 6 April 2015. http:// www.jpalliativecare.com/palliativeprograms.asp.

Janakiram, C., and S.J. Gardens. 2014. Knowledge, attitudes and practices related to healthcare ethics among medical and dental postgraduate students in south India. Indian Journal of Medical Ethics 11(2): 99-104.

Oregon Health Authority. 2015. "The Oregon Death with Dignity Act. Oregon Revised Statutes.” Accessed 6 April 2015. https://public.health.oregon.gov/ProviderPartnerResources/EvaluationResearch/ DeathwithDignityAct/Pages/ors.aspx.

Pati, S., A. Sharma, and S. Zodpey. 2014. Teaching of public health ethics in India: a mapping exercise. Indian Journal of Medical Ethics 11(3): 185-90.

Raj, E.H. 2012. The changing scenario in oncologist-patient communication: We need to adapt... ...the Cherny article reviewed. Oncology 26(1): 48-55.

Raja, K. 2007. Patients' perspectives on medical information: results of an informal survey. Indian Journal of Medical Ethics 4(1): 16-7. 
Sanwal, A.K., S. Kumar, P. Sahni, and S. Nundy. 1996. Informed consent in Indian patients. Journal of the Royal Society of Medicine 89(4): 196-8.

Schneiderman, L.J., T. Gilmer, H.D. Teetzel, D.O. Dugan, J. Blustein, R. Cranford, K.B. Briggs, G.I. Komatsu, P. Goodman-Crews, F. Cohn, and E.W. Young. 2003. Effect of ethics consultations on nonbeneficial life-sustaining treatments in the intensive care setting: a randomized controlled trial. JAMA 290(9): 1166-72.

Seetharam, S., and R. Zanotti. 2009. Patients' perceptions on healthcare decision making in rural India: a qualitative study and ethical analysis. Journal of Clinical Ethics 20(2): 150-7.

Senarath, U., and N.S. Gunawardena. 2009. Women's autonomy in decision making for health care in South Asia. Asia-Pacific Journal of Public Health 21(2): 137-143.

Subramanian, T., A.K. Mathai, and N. Kumar. 2013. Knowledge and practice of clinical ethics among healthcare providers in a government hospital, Chennai. Indian Journal of Medical Ethics 10(2): 96-100.

Tronto, J.C. 1993. Moral boundaries. A political argument for an ethic of care. New York: Routledge.

Widdershoven, G.A. 2002. Beyond autonomy and beneficence: the moral basis of euthanasia in the Netherlands. Ethical Perspectives 9(2-3): 96-102.

World Bank. 2015. "Health expenditure, public (\% of GDP)" Accessed January 22. http://data.worldbank.org/ indicator/SH.XPD.PUBL.ZS/countries?display=default.

Yousuf, R.M., A.R. Fauzi, S.H. How, A.G. Rasool, and K. Rehana. 2007. Awareness, knowledge and attitude toward informed consent among doctors in two different cultures in Asia: a cross-sectional comparative study in Malaysia and Kashmir, India. Singapore Medical Journal 48(6): 559-65. 